\title{
Time-resolved neutron/gamma-ray data acquisition for in situ subsurface planetary geochemistry
}

\author{
J.G. Bodnarik ${ }^{\mathrm{a}, \mathrm{b}, *}$, D.M. Burger ${ }^{c}$, A. Burger ${ }^{\text {d }}$, L.G. Evans ${ }^{\mathrm{e}}$, A.M. Parsons ${ }^{\text {a }}$, J.S. Schweitzer ${ }^{\text {f }}$, R.D. Starr ${ }^{\text {, }}$, \\ K.G. Stassun ${ }^{\mathrm{b}}$ \\ a NASA Goddard Space Flight Center, 8800 Greenbelt Road, Code 691, Greenbelt, MD 20771, USA \\ ${ }^{\mathrm{b}}$ Vanderbilt University, Department of Physics and Astronomy, VU Station B \#1807, Nashville, TN 37235, USA \\ ${ }^{c}$ Vanderbilt University, VU Station B \#351679, 2301 Vanderbilt Place, Nashville, TN 37235-1679, USA \\ ${ }^{\mathrm{d}}$ Fisk University, Department of Physics, 240 W.E.B. DuBois Hall, 1000 17th Avenue N., Nashville, TN 37208, USA \\ e Computer Sciences Corporation, 7900 Harkins Road, Lanham, MD 20706, USA \\ ${ }^{\mathrm{f}}$ University of Connecticut, Department of Physics, Storrs, CT 06269-3046, USA \\ ${ }^{g}$ Catholic University of America, Institute for Astrophysics and Computational Sciences, DC 20064, USA
}

\section{A R T I C L E I N F O}

\section{Article history:}

Received 3 August 2012

Received in revised form

19 December 2012

Accepted 20 December 2012

Available online 27 December 2012

Keywords:

Elemental analysis

Pulsed neutron generator

Time-tagged data acquisition

Optimized time-gating

Time-dependent neutron and gamma-ray detection

\begin{abstract}
A B S T R A C T
The current gamma-ray/neutron instrumentation development effort at NASA Goddard Space Flight Center aims to extend the use of active pulsed neutron interrogation techniques to probe the subsurface elemental composition of planetary bodies in situ. Previous NASA planetary science missions, that used neutron and/or gamma-ray spectroscopy instruments, have relied on neutrons produced from galactic cosmic rays. One of the distinguishing features of this effort is the inclusion of a high intensity $14.1 \mathrm{MeV}$ pulsed neutron generator synchronized with a custom data acquisition system to time each event relative to the pulse. With usually only one opportunity to collect data, it is difficult to set $a$ priori time-gating windows to obtain the best possible results. Acquiring time-tagged, event-byevent data from nuclear induced reactions provides raw data sets containing channel/energy, and event time for each gamma ray or neutron detected. The resulting data set can be plotted as a function of time or energy using optimized analysis windows after the data are acquired. Time windows can now be chosen to produce energy spectra that yield the most statistically significant and accurate elemental composition results that can be derived from the complete data set. The advantages of post-processing gamma-ray time-tagged event-by-event data in experimental tests using our prototype instrument will be demonstrated.
\end{abstract}

Published by Elsevier B.V.

\section{Introduction}

The objective of the current gamma-ray/neutron instrumentation development at NASA Goddard Space Flight Center (GSFC) is

\footnotetext{
Abbreviations: PNG, Pulsed Neutron Generator; PING, Pulsing In situ with Neutrons and Gamma rays; GSFC, Goddard Space Flight Center; MESSENGER, MErcury Surface, Space ENvironment, GEochemistry and Ranging; LRO, Lunar Reconnaissance Orbiter; NEAR, Near Earth Asteroid Rendezvous; GRC, Galactic Cosmic Rays; MCNPX, Monte Carlo N-Particle eXtended; DSA, Digital Signal Analyzer; PHA, Pulse Height Analysis; TLIST, Time-stamped LIST; HPGe, High Purity Germanium; GGAO, Goddard's Geophysical and Astronomical Observatory; SE, Single Escape (refers to HPGe spectra for pair production)

* Corresponding author at: NASA Goddard Space Flight Center, 8800 Greenbelt Road, Code 691, Greenbelt, MD 20771, USA. Tel.: +1 808-896-6759; fax: +13012861683.

E-mail addresses: julia.g.bodnarik@nasa.gov, julie.g.bodnarik@gmail.com (J.G. Bodnarik), dan.burger@vanderbilt.edu (D.M. Burger), aburger@fisk.edu (A. Burger), larry.g.evans@nasa.gov (L.G. Evans), ann.m.parsons@nasa.gov (A.M. Parsons), schweitz@phys.uconn.edu (J.S. Schweitzer), richard.d.starr@nasa.gov (R.D. Starr), keivan.stassun@vanderbilt.edu (K.G. Stassun).
}

to use active pulsed neutron interrogation techniques to determine in situ the subsurface bulk elemental abundances of planetary bodies. To date, all the planetary science missions that have included both neutron and gamma-ray instruments have made remote sensing measurements from orbit or during close fly-by encounters with a planetary body (e.g. Lunar Prospector [1], Mars Odyssey [2,3], Dawn [4], MESSENGER [5], NEAR [6], and LRO $[7,8])$. The excitation sources for these remote sensing measurements have necessarily been limited to the high-energy (fast) neutrons that are produced when Galactic Cosmic Rays (GCR) interact with planetary materials. Although GCR-generated fast neutron rates change with the 11-year solar cycle, they occur at a constant rate for the durations of these measurements. Measured gamma-ray spectra contain all of the gamma-ray lines from each of the gamma ray-producing interactions of neutrons with the planetary material. Gamma-ray spectra thus include peaks resulting from inelastic scattering of fast neutrons, thermal neutron capture, delayed activation and natural radioactivity. However, the low gamma-ray flux, the large number of peaks and the high spectral background result in peak interferences, 
misidentifications and increased uncertainty in the reported gamma-ray results. These difficulties can be substantially lessened for in situ measurements of a landed instrument package that includes a Pulsed Neutron Generator (PNG) as the excitation source. A PNG can produce fast neutrons at $\sim 100$ times greater rate than GCR interactions, resulting in significantly reduced measurement times for equivalent sensitivity. A PNG can also produce $14.1 \mathrm{MeV}$ neutrons in relatively short bursts with an adjustable neutron pulse period and width. With the production of the high-energy neutrons restricted to the duration of the burst, the gamma rays that result from the inelastic scattering of these fast neutrons will also occur only during the time of the burst. Between each burst, the planetary material moderates the fast neutrons so that the gamma rays are largely produced by thermal neutron capture. After most of the thermal neutrons have been absorbed, the gamma rays resulting from delayed activation and natural radioactivity become visible. Separating the gamma rays by their detection time relative to a PNG pulse results in lower background and a substantial reduction in peak interferences, while capturing essentially all of the gamma rays due to a particular type of reaction. Separating gamma ray spectra by physical process minimizes the systematic effects from interfering peaks and provides improved precision and accuracy in the peak analysis directly resulting in more precise elemental concentration measurements. We have previously shown [9] that significant improvements in precision can be obtained using properly chosen time windows for time-gated coincidence data acquisition methods. Here we report the increased benefits of using this time-tagged event-by-event data.

On Earth, it is possible to adjust the PNG pulse period and width as well as the coincident data acquisition window timing parameters for an optimum analysis of a sample because one usually has a general idea of the sample's bulk composition and its properties with regard to neutron and gamma ray transport. Even without this knowledge, multiple measurements using adjusted parameters are usually possible. So it is often simple and sufficient to use coincidence data acquisition methods with a limited number of fixed time gates for these ground-based experiments on Earth. However, one rarely has the luxury of repeating measurements at a particular point on another planet. When making in situ measurements on a planetary body, there is often a large unknown regarding its composition, especially with regard to elements that affect the neutron and gamma-ray time dependence. For planetary science applications it would be very difficult to make multiple measurements at a variety of different timing conditions with sufficient statistics to determine the optimum timing parameters. The optimal timing parameters largely depend on neutron transport properties that are governed by effects that vary by location such as elemental composition, hydrogen content, density and subsurface layering geometries. By the time one has determined what the proper time gating should be, the mission may be over, or, in the case of a rover mission, the rover may have already left the region where the earlier data were obtained.

This type of problem has been addressed in early NASA Apollo gamma-ray experiments [10] as well as in other scientific fields such as radioanalytical chemistry applications [11] by accumulating data on an event-by-event basis where the energy and measurement time is recorded for every event detected during the data acquisition time. When data are accumulated in an event-by-event mode that includes event times, one can analyze the data after the measurement has been made (post-processing) to determine the optimum time windows for spectral data analysis. Although event-by-event data acquisition leads to large raw data files, it makes it possible to perform the optimal spectral analysis without requiring repeated measurements. Until data transmission capabilities are improved, one approach is to take initial data in event-by-event mode to determine optimum location for window boundaries and take the rest of the data at this position in long accumulations. New event-by-event data would be taken when the instrument is moved to a new location.

\subsection{The Probing In situ with Neutrons and Gamma-rays (PING) instrument}

Our group at NASA/GSFC is currently developing the Probing In situ with Neutrons and Gamma-rays (PING) instrument for planetary in situ bulk elemental composition measurements [9] by leveraging both well-established oil well and scientific logging techniques [12] and remote sensing planetary gamma-ray spectroscopy techniques. PING employs a $14.1 \mathrm{MeV}$ pulsed neutron generator to excite materials at and below a planetary surface and utilizes the penetrating nature of these fast neutrons and gamma rays to probe the subsurface soil composition over a $1 \mathrm{~m}^{2}$ area and down to depths of $10-100 \mathrm{~cm}$. A gamma-ray spectrometer measures the resulting inelastic-scattering, capture, and delayedactivation gamma rays emitted by the excited elements, as well as gamma rays emitted from natural radioactive decay, that emerge from the planetary surface. Neutron detectors measure the number of the epithermal and thermal neutrons that reach the surface as a function of time relative to the initiation of each highenergy neutron pulse. PING can measure a wide range of elements (e.g. C, H, O, P, S, Si, Na, Ca, Ti, Fe, Al, Cl, Mg, Mn, K, Th, and U), depending on their detectable abundance in the planetary material and interference of any neighboring gamma-ray peaks. To illustrate an example application, PING is shown in Fig. 1 attached to the underside of a planetary rover.

PING gamma-ray and neutron data are acquired using custom software to control the digital signal analyzer electronics and synchronize time-tagged event-by-event data acquisition with the start of each PNG burst. These data will be coupled with MCNPX [13] computer simulations in future work to allow us to quantitatively determine the bulk elemental composition of the subsurface material for any solid body in the solar system. The MCNPX calculations allow an exact statistical calculation of both the energy and the time of a gamma-ray event detected in a detector. The calculations take into account all the details of neutron production and transport, all possible nuclear reactions on all elements present in the material, all possible gamma rays that can be produced and their transport and detection at a specific point in space by a particular detector. Thus, the Monte

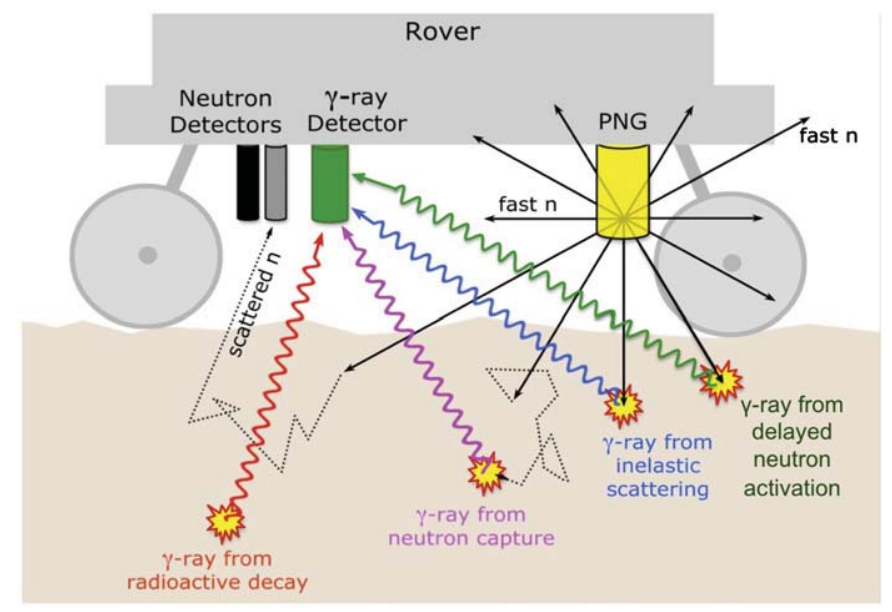

Fig. 1. Illustration of PING. The instrument is mounted on the underside of a planetary surface rover. Also shown are the different nuclear processes that produce the gamma rays and scattered neutrons that are detected at the surface. 


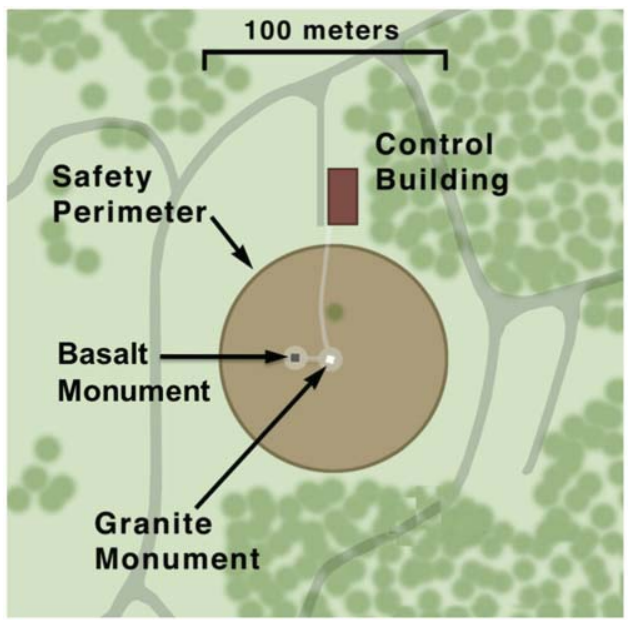

Fig. 2. Aerial view of GGAO. This schematic of the outdoor gamma ray and neutron instrumentation testing facility shows the operations control building as well as the $46 \mathrm{~m}$ diameter safety perimeter surrounding the two existing $1.8 \mathrm{~m} \times 1.8 \mathrm{~m} \times 0.9 \mathrm{~m}$ granite and basalt monuments.

Carlo calculations provide a direct relationship between peak counts and elemental concentrations, limited only by the count rate uncertainty and the calculation uncertainties (generally less than $1 \%)$.

\subsection{Outdoor neutron-gamma ray instrument test site}

We are testing the capabilities of our PING instrument prototypes at a unique outdoor gamma ray and neutron instrumentation testing facility located at Goddard's Geophysical and Astronomical Observatory (GGAO) near the Goddard main campus. A schematic view of the test site is shown in Fig. 2. This test facility allows us to operate PING on top of either of two large, well-characterized granite and basalt monuments, each $1.8 \mathrm{~m} \times 1.8 \mathrm{~m} \times 0.9 \mathrm{~m}$ in size. Activation Laboratories Ltd. in Ancaster, Ontario, Canada, has independently measured the full elemental compositions of these Concord Gray Granite and Columbia River Basalt materials to the ppm level. PING is remotely operated from a building more than $75 \mathrm{~m}$ from the monuments due to the radiation hazard from the PNG's $14 \mathrm{MeV}$ neutrons. Underground power and communications lines connect the operations building to the test monuments. Details of the specific PING measurements are given in Section 3.1 and further information about the test facility can be found in $[14,15]$.

\subsection{Using TLIST data to improve PING elemental composition measurements}

A Canberra Lynx Digital Signal Analyzer (DSA) is used to acquire data from each gamma-ray and neutron detector used for a PING measurement. While the Lynx DSA hardware [16] features multiple data acquisition modes, including coincidencegated Pulse Height Analysis (PHA) and event-by-event Timestamped LIST (TLIST) mode, operation of the Lynx DSAs in TLIST mode required the development of custom software. In this paper, we describe both the acquisition of TLIST data using our custom MultiScan software [17] and the post-processing of our data that allows us to:

(1) Use optimized timing windows to separate the data into distinct gamma-ray spectra resulting from either (a) inelastic scattering, during the neutron pulse, (b) thermal neutron capture, between neutron pulses, or (c) delayed activation and natural activity events visible just before the next fast neutron pulse. This separation allows us to more accurately identify gamma ray lines and more precisely measure gamma-ray net peak areas;

(2) isolate a particular energy line from a gamma-ray spectrum and observe its intensity time profile with respect to the PNG pulse to aid in nuclide identification, since the raw event-byevent data preserves the time and energy information for each gamma-ray event, which is impossible to do with preset time windows that only preserve gamma-ray energy information; and

(3) extract gamma-ray data to optimize the timing windows needed to look for specific elements in different environments and to obtain the optimum precision for the analyzed peak intensities.

\section{The TLIST data acquisition technique}

Analyzing individual gamma-ray peaks in a traditional PHA energy spectrum can be challenging due to both interfering lines and the background continuum resulting from multiple processes. We reduce these effects and obtain higher gamma-ray line sensitivity with increased signal-to-noise by recording gamma-ray time and energy in an event-by-event mode synchronized to the start of each PNG pulse. We use our custom MultiScan software and the Canberra Lynx DSA in TLIST mode to record the energy and time (temporal resolution $0.1 \mu \mathrm{s}$ ) of each event detected during a PNG pulse cycle. As discussed in Section 1, we obtain a master data set that is not limited to predetermined coincidence timing gates set for specific nuclear processes. This master data set can be sliced in many ways without loss of information or requiring additional measurements with different data acquisition window settings. Fig. 3(a) and (b) illustrates the results of our post-processing of TLIST gamma-ray data for various timing windows. The sharp lines shown in this figure are merely used to demonstrate how one can take advantage of time-slicing gamma-ray data. An important benefit of this technique is that for specific gamma-ray peaks, different windows may be selected than those that apply to the bulk of the data. For example, a delayed activation peak that does not interfere with a capture peak can have an analysis window that starts well before the bulk of the capture gamma rays have disappeared.

Fig. 3(a) is an illustration of the PNG fast neutron pulse train and the intra-pulse location of the different timing windows needed to separate the gamma rays that result from the inelastic scattering, thermal neutron capture, delayed activation and natural radioactivity processes. Fig. 3(b) is an illustration of the differences in the resulting energy and intensity of the gamma ray lines and background for each of these separated spectra.

\subsection{Custom multiscan data acquisition software}

Lynx DSA data acquisition can be performed using either the Lynx web-based interface or the Genie 2000 software package [16], both available from Canberra Industries. Although the Lynx DSA hardware offers the required TLIST mode, neither of these software options provides the flexibility and all of the capabilities we need for our specific instrument application. The MultiScan software, designed specifically for our project, allows us to (1) acquire data in TLIST mode while synchronized to the PNG pulse, (2) save data in ASCII format, (3) analyze TLIST data for an unlimited number of time windows, and (4) perform multiple consecutive data acquisitions while maintaining the Lynx graphical analysis and configuration features. The program also provides basic data analysis tools for both PHA and TLIST scans, and offline TLIST data post-processing 
a

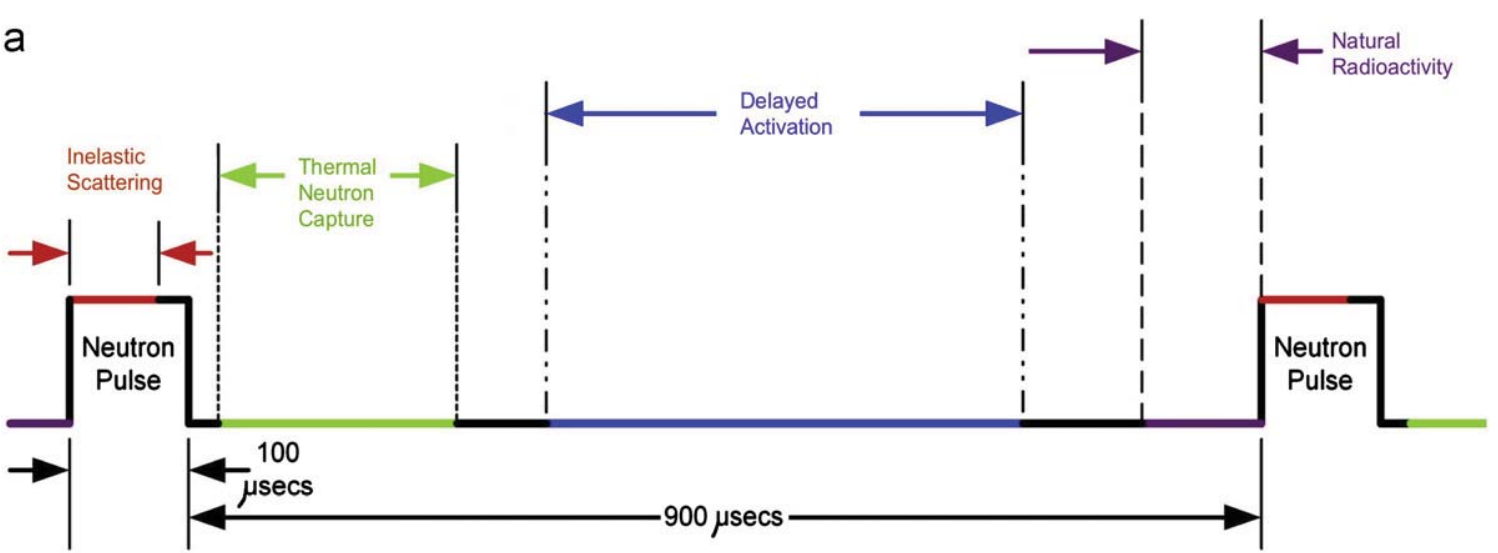

b
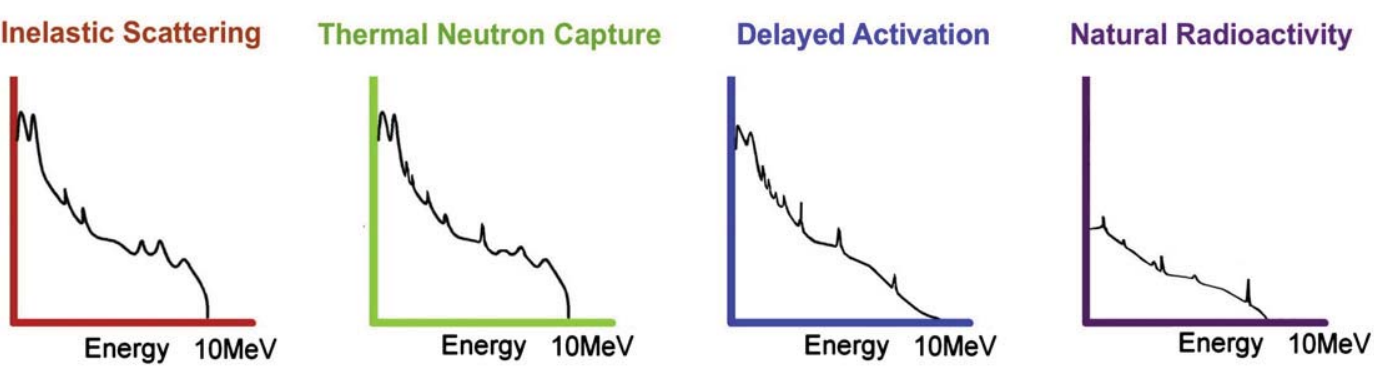

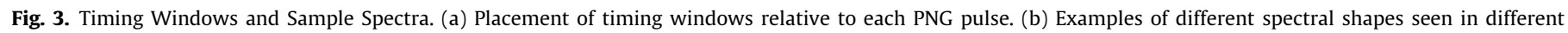
timing windows.

time-slicing tools, as well as a diagnostic feature for monitoring the operating parameters within the Lynx DSA [18].

The MultiScan software was written in Java, since we needed to make the code cross-platform and easy to understand so that others can make changes to the code when necessary. When starting a new data acquisition or scan, the user can specify which of the multiple Lynx DSAs to perform the scan, the acquisition mode (PHA or TLIST), the file format to save the data (Canberra CNF file, ASCII text, or both), how many consecutive scans to perform, and the duration of each scan (in either live time or true time). Settings can be modified quickly and easily within the software. The data are both written to a file and presented in a large display window with multiple data visualization features.

\subsection{TLIST data analysis techniques}

We use the MultiScan software with Lynx DSAs to acquire TLIST data for gamma-ray and neutron detectors with the start of a data acquisition synchronized with the start of a PNG pulse. Synchronization of the PNG and DSA clocks insures the accuracy of these event times over multi-hour data acquisition runs. Our basic post-processing procedure for the individual event-by-event data files is to take the modulus of the absolute times for the detected events with respect to the known PNG pulse period to derive the time of each event relative to the neutron pulse. The next step is to put all of the files for a given experiment on the same time base. The result is a master data set of energies and relative event times that can be "sliced" in any number of ways. Slicing the data in time means establishing the boundary between times where different nuclear processes dominate. The result is separate gamma-ray spectra for the specific processes that have the event statistics characteristic of the total acquisition time. Slicing the data in energy means establishing energy boundaries around spectral features whose time profile one wishes to study. After generating this master data set with energy and relative time values, we can analyze our gamma ray and neutron data to infer the bulk elemental composition, density, and subsurface layering of planetary bodies.

\section{Experiments and results with TLIST data}

We performed PING experiments using a pulsed neutron generator, gamma ray and neutron detectors on a meter-sized basalt monument. The TLIST data acquired and analyzed in this section only represents $6.33 \mathrm{~h}$ of data acquisition with a fixed neutron pulse with a width of $100 \mu$ s and a pulse period of $1000 \mu$ s. The results of TLIST data acquisition and post-processing presented will demonstrate the improved precision and reduced systematic errors that can be achieved as compared with pre-assigned acquisition windows from a presumed knowledge of elemental composition.

\subsection{Experiment description}

During these experiments, we acquired data using a Lynx DSA connected to an n-type Ortec GMX Series HPGe portable coaxial detector system (crystal diameter $=53.2 \mathrm{~mm}$ and crystal length $=69.5 \mathrm{~mm}$ ) and a $14 \mathrm{MeV}$ Deuterium-Tritium Thermo Fisher MP320 portable PNG [19] positioned on top of our Columbia River basalt monument, as shown in Fig. 4.

The Lynx DSA reading out the HPGe detector was connected directly to the PNG to synchronize the start of each data acquisition run with the start of a neutron pulse. The PNG beam current, high voltage, frequency, and duty factor were set to $60 \mu \mathrm{A}, 50 \mathrm{kV}$, $1 \mathrm{kHz}$, and 10\% respectively. At these settings, the PNG produced a neutron pulse width, pulse period, energy, and rate of $100 \mu \mathrm{s}$, $1000 \mu \mathrm{s}, 14 \mathrm{MeV}$, and $3 \times 10^{7} \mathrm{n} / \mathrm{s}$ respectively.

\subsection{Gamma-ray peak separation using TLIST data analysis}

Gamma-ray line identification problems can be lessened with the PING instrument by taking advantage of the pulsed nature of 
the in situ neutron source synchronized with the data acquisition system, particularly if the neighboring energies originate from reactions having different time delays relative to the production of the neutron. Naturally, different reactions that occur at the same time, such as prompt $\left(n, n^{\prime}\right),(n, p)$ and $(n, \alpha)$ reactions that all require high energy neutrons cannot be separated from each other by selecting different analysis times.

Fig. 5 is a plot of four different gamma-ray spectra for a 6.33-hr live time acquisition with the PING instrument using a HPGe detector on the basalt monument, consisting of: (1) a total gamma-ray spectrum (in black) including all neutron-nuclei gamma-ray processes; (2) an inelastic gamma-ray spectrum (in red) created by only selecting gamma-ray events during the PNG pulse for $t=20-100 \mu \mathrm{s}$; (3) a neutron capture gamma-ray spectrum (in green) created by only selecting gamma-ray events after the PNG pulse for $t=150-650 \mu \mathrm{s}$; and (4) a delayed activation and natural activity gamma-ray spectrum (in purple) created by only selecting gamma-ray events for $t=650-999 \mu \mathrm{s}$. Note that, as expected, different gamma-ray lines appear in these spectra. Our technique thus allows us to isolate gamma-ray events for specific interactions from a single element without accumulating excessive background when the peaks are not actually present.

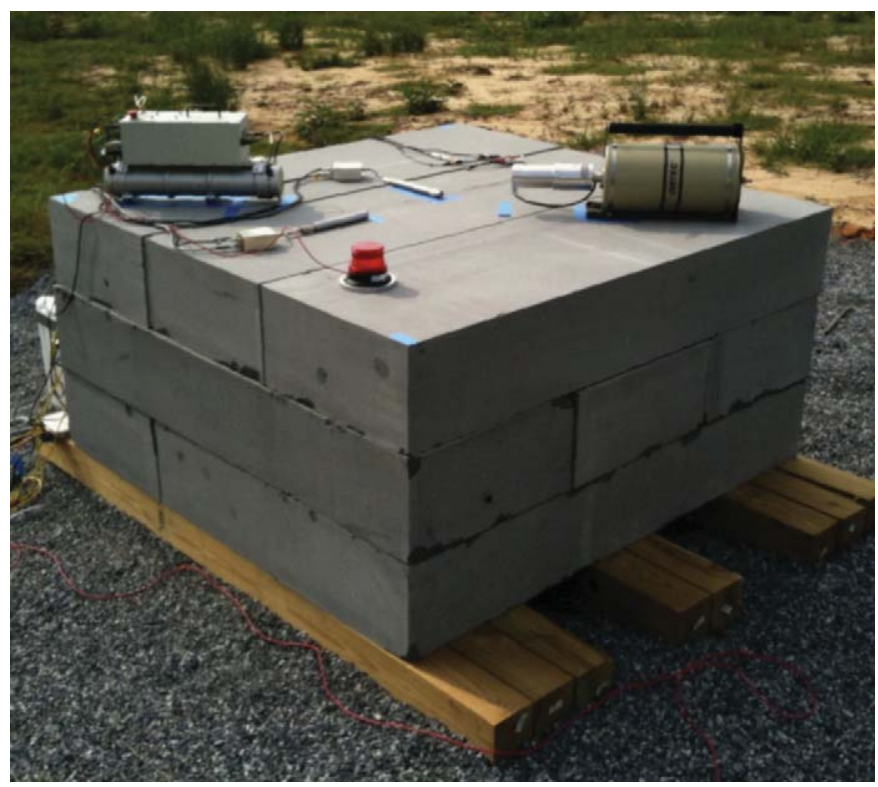

Fig. 4. PING Experiment Set-up. PING deployed for measurements on top of the basalt monument. The PNG is on the left, the HPGe detector is on the right, and ${ }^{3}$ He detectors are between them. The data acquisition electronics are situated behind the basalt and are not visible in this photo.
Even if a better energy resolution detector like HPGe is used, gamma-ray line identification can still be challenging, due to multi-element neutron-nuclei interactions that produce gamma rays at the same energy but from different elements. Table 1 lists examples of gamma-ray line energies and their possible sources from neutron-nuclei interactions with different elements, demonstrating how multiple elements can contribute to the same line energy. (Note that the first entry in Table 1 contains two gamma ray lines at slightly different energies. They are grouped together because under many circumstances, they cannot be separated.)

Problems with interfering lines can be dealt with by examining the time profile of the individual gamma-ray lines. Fig. 6(a) is an example of a $6.33 \mathrm{~h}$ summed HPGe gamma-ray spectrum taken with PING instrument on top of the basalt monument. In this spectrum there are many gamma ray lines that are clearly interfering with one another such as, the Doppler broadened ${ }^{27} \mathrm{Al}\left(\mathrm{n}, \mathrm{n}^{\prime} \gamma\right),{ }^{1} \mathrm{H}(\mathrm{n}, \gamma),{ }^{24} \mathrm{Na}(\mathrm{SE})$, the Doppler broadened ${ }^{24} \mathrm{Mg}\left(\mathrm{n}, \mathrm{n}^{\prime} \gamma\right)$, and the ${ }^{30} \mathrm{Si}\left(\mathrm{n}, \mathrm{n}^{\prime} \gamma\right)$. One way to distinguish ${ }^{27} \mathrm{Al}\left(\mathrm{n}, \mathrm{n}^{\prime} \gamma\right)$ and the ${ }^{1} \mathrm{H}(n, \gamma)$ gamma ray lines is by plotting the net peak area of the unresolved spectral feature in Fig. 6(a) as a function of time, as shown in Fig. 6(b), to distinguish which line is present. Fig. 6(b) shows the time histograms of the net peak areas for the $2211 \mathrm{keV}$ ${ }^{27} \mathrm{Al}\left(\mathrm{n}, \mathrm{n}^{\prime} \gamma\right)$ and the $2223 \mathrm{keV}{ }^{1} \mathrm{H}(\mathrm{n}, \gamma)$ gamma ray lines. The time histograms are the gamma-ray count rates per $10 \mu$ s time interval and demonstrate that one can distinguish between and separate interfering lines by nuclear process to improve both the peak identification and the measurement precision.

\subsection{Improved gamma-ray measurement precision}

By reducing the background, separating a gamma-ray spectrum by nuclear process improves the overall gamma-ray line

Table 1

The same gamma-ray line energies can come from neutron-nuclei interactions with different elements.

\begin{tabular}{ll}
\hline Gamma-ray lines (keV) & Possible sources of neutron-nuclei interactions \\
\hline $844-847$ & A, B, C, D, E \\
1014 & A, D \\
1779 & F, G, H \\
1811 & B, C, E, I* \\
2211 & A \\
6129 & J, K
\end{tabular}

Key: A: ${ }^{27} \mathrm{Al}\left(\mathrm{n}, \mathrm{n}^{\prime} \gamma\right){ }^{27} \mathrm{Al}, \mathrm{B}:{ }^{56} \mathrm{Fe}\left(\mathrm{n}, \mathrm{n}^{\prime} \gamma\right){ }^{56} \mathrm{Fe}, \mathrm{C}:{ }^{56} \mathrm{Fe}(\mathrm{n}, \mathrm{p}){ }^{56} \mathrm{Mn}(\beta){ }^{56} \mathrm{Fe}, \mathrm{D}:{ }^{26} \mathrm{Mg}$ $(\mathrm{n}, \gamma){ }^{27} \mathrm{Mg}(\beta){ }^{27} \mathrm{Al}, \mathrm{E}:{ }^{55} \mathrm{Mn}(\mathrm{n}, \gamma){ }^{56} \mathrm{Mn}(\beta){ }^{56} \mathrm{Fe}, \mathrm{F}:{ }^{28} \mathrm{Si}\left(\mathrm{n}, \mathrm{n}^{\prime} \gamma\right){ }^{28} \mathrm{Si}, \mathrm{G}:{ }^{28} \mathrm{Si}(\mathrm{n}, \mathrm{p})$ ${ }^{28} \mathrm{Al}(\beta){ }^{28} \mathrm{Si}, \mathrm{H}:{ }^{27} \mathrm{Al}(\mathrm{n}, \gamma){ }^{28} \mathrm{Al}(\beta){ }^{28} \mathrm{Si}, \mathrm{I}^{*}:{ }^{26} \mathrm{Mg}\left(\mathrm{n}, \mathrm{n}^{\prime} \gamma\right){ }^{26} \mathrm{Mg}, \mathrm{J}:{ }^{16} \mathrm{O}\left(\mathrm{n}, \mathrm{n}^{\prime} \gamma\right){ }^{16} \mathrm{O}, \mathrm{K}:$ ${ }^{16} \mathrm{O}(\mathrm{n}, \mathrm{p}){ }^{16} \mathrm{~N}(\beta){ }^{16} \mathrm{O}$.

* Alternate source: ${ }^{27} \mathrm{Al}(\mathrm{n}, \mathrm{d} \gamma){ }^{26} \mathrm{Mg}$

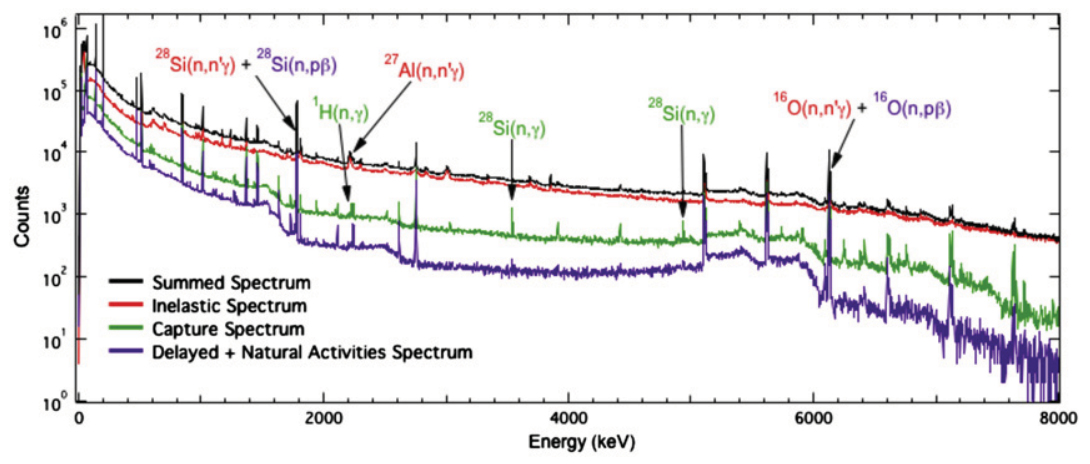

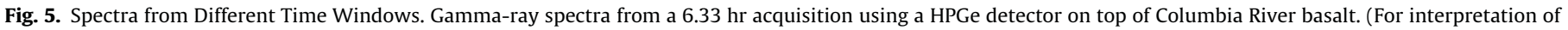
the references to color in this figure legend, the reader is referred to the web version of this article.) 
measurement precision. As seen in Table 2, listing the total number of peak counts in an energy peak for different time windows, many of the time-gated inelastic scattering and capture lines show improved precision as compared with the same lines in the summed spectrum. The 3539 and $4934 \mathrm{keV}{ }^{28} \mathrm{Si}(\mathrm{n}, \gamma)$ capture lines show improved precision resulting from timegated analysis. The precision of these Si lines in the summed spectrum, representing results without time slicing, is $8.3 \%$ and 16.9\%. These same Si lines show improved precision $(7.3 \%$ and $9.2 \%$ ) in the thermal neutron capture spectrum obtained from the removal of the gamma-ray background due to inelastic scattering.
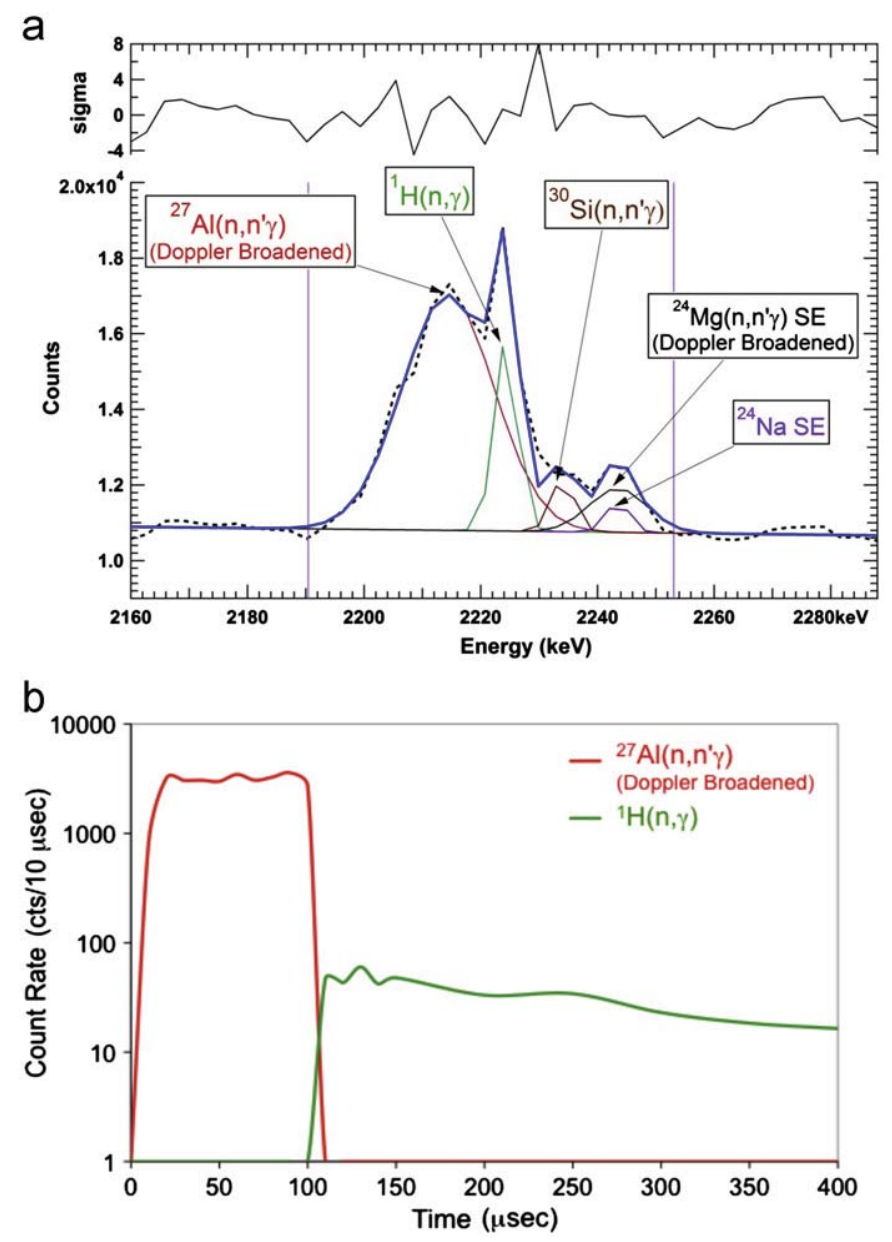

Fig. 6. Spectral Feature and Time Distribution. (a) A portion of the non-time sliced $6.33 \mathrm{~h}$ gamma ray energy histogram from PING data taken on the bare basalt monument. (b) Time histogram showing how one can get better precision on the net peak area of each line, shown in Table 2, by analyzing their respective energy histograms during different time slices during the PNG pulse period.
A similar but somewhat smaller improvement is seen for the $2211 \mathrm{keV}^{27} \mathrm{Al}\left(\mathrm{n}, \mathrm{n}^{\prime} \gamma\right)$ inelastic line.

An interesting situation is observed for the $1779 \mathrm{keV}$ ${ }^{28} \mathrm{Si}\left(\mathrm{n}, \mathrm{n}^{\prime} \gamma\right)$ and $6129 \mathrm{keV}{ }^{16} \mathrm{O}\left(\mathrm{n}, \mathrm{n}^{\prime} \gamma\right)$ inelastic lines shown in Table 2 . These gamma rays are also produced in the other two spectra by delayed activation reactions (see Table 1 ). Therefore, the 1779 and $6129 \mathrm{keV}$ gamma ray lines in the summed spectrum have a better statistical precision of $0.48 \%$ and $1.10 \%$ as compared to $1.00 \%$ and $1.67 \%$ (inelastic spectrum) and $0.52 \%$ and $1.42 \%$ (delayed activation spectrum), because there are more counts in the summed spectrum.

Gamma-ray peaks will obviously have the best statistical precision if the counts recorded at all times are summed. However, when there are times where counts are produced by more than a single reaction on a single element, there is no longer a linear relationship between the counts in the peak and the concentration of a single element. To get the most accurate result for an element's concentration, it is thus necessary to remove all of the counts measured at times when they can be produced by multiple reactions or by different elements (see Table 1 ).

While this procedure may reduce the statistical precision somewhat, it significantly improves the accuracy, which would otherwise be deteriorated by assigning counts to the wrong element. This problem can be seen when looking at the data for the $1779 \mathrm{keV}$ peak in Table 2. One would like to have the $1779 \mathrm{keV}$ peak that occurs during the high-energy neutron pulse be only due to silicon. However, there is also peak at the same energy that is due to the delayed activity of aluminum. Since delayed activity peaks are present at all times, if these counts were not subtracted from the peak measured during the highenergy neutron pulse, the derived elemental concentration would be much too high. This effect can be seen in the data in Table 2 .

To relate measured peak count rates to elemental concentrations, we need to be certain that our peak areas can be related to a single specific reaction. Then known quantities, such as reaction cross-section and atomic concentration will tell us how many counts correspond to what weight percent. Unfortunately, the measured counts in the peak also depend on neutron and gammaray transport and the detector efficiency. The effects of these processes can be calculated with the Monte Carlo code. For Si and $\mathrm{O}$ (assay concentration ratio $=0.52$ ), the transport and detector efficiency yield a ratio of 4.89 for the concentrations provided by the chemical assay. However, we need to be sure the measured peak areas we use reflect only the counts due to a single reaction.

If we did no time gating and assumed that the measured 1779 peak was only due to silicon, we would have $90480+/-0.48 \%$ counts and for the measured oxygen peak at $6129 \mathrm{keV}$, we would have $19920+/-1.1 \%$ counts. The Si/O ratio would then be about 4.5 . Dividing this value by the efficiency ratio, 4.89 , provides an experimental $\mathrm{Si} / \mathrm{O}$ ratio of 0.92 , significantly greater than the 0.52 concentration ratio from the chemical assay.

Table 2

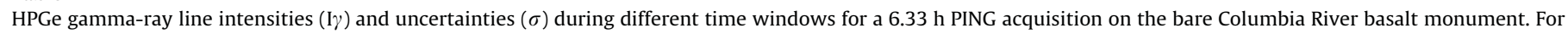

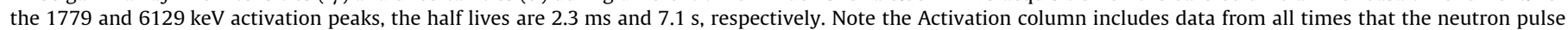

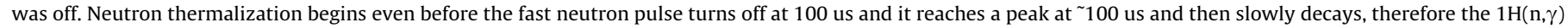
$2223 \mathrm{keV}$ gamma-ray line appears in both the inelastic scattering and thermal neutron capture windows due to the time windows selected for these processes.

\begin{tabular}{|c|c|c|c|c|c|c|c|c|c|c|c|}
\hline \multirow[t]{2}{*}{ Energy (keV) } & \multicolumn{2}{|c|}{ Window summed data } & \multicolumn{3}{|c|}{ Inelastic scattering window } & \multicolumn{3}{|c|}{ Thermal neutron capture window } & \multicolumn{3}{|l|}{ Activation } \\
\hline & $\mathrm{I} \gamma(\mathrm{cts})$ & $\sigma(\%)$ & ID & $\mathrm{I} \gamma(\mathrm{cts})$ & $\sigma(\%)$ & ID & $\mathrm{I} \gamma(\mathrm{cts})$ & $\sigma(\%)$ & ID & $\mathrm{I} \gamma(\mathrm{cts})$ & $\sigma(\%)$ \\
\hline 1779 & 90480 & 0.48 & ${ }^{28} \mathrm{Si}\left(\mathrm{n}, \mathrm{n}^{\prime} \gamma\right)$ & 31730 & 1.0 & & & & ${ }^{28} \mathrm{Si}(\mathrm{n}, \mathrm{p}){ }^{28} \mathrm{Al}{ }^{27} \mathrm{Al}(\mathrm{n}, \gamma){ }^{28} \mathrm{Al}$ & 57980 & 0.52 \\
\hline 2211 & 24310 & 1.55 & ${ }^{27} \mathrm{Al}\left(\mathrm{n}, \mathrm{n}^{\prime} \gamma\right)$ & 23760 & 1.5 & & & & & & \\
\hline 2223 & 1892 & 16.1 & ${ }^{1} \mathrm{H}(\mathrm{n}, \gamma)$ & 967 & 14.5 & ${ }^{1} \mathrm{H}(\mathrm{n}, \gamma)$ & 887 & 7.4 & & & \\
\hline 3539 & 1154 & 8.3 & & & & ${ }^{28} \mathrm{Si}(\mathrm{n}, \gamma)$ & 1158 & 7.3 & & & \\
\hline 4934 & 1472 & 16.9 & & & & ${ }^{28} \mathrm{Si}(\mathrm{n}, \gamma)$ & 1151 & 9.2 & & & \\
\hline 6129 & 19920 & 1.1 & ${ }^{16} \mathrm{O}\left(\mathrm{n}, \mathrm{n}^{\prime} \gamma\right)$ & 10900 & 1.67 & & & & ${ }^{16} \mathrm{O}(\mathrm{n}, \mathrm{p}){ }^{16} \mathrm{~N}$ & 9087 & 1.42 \\
\hline
\end{tabular}


Even rudimentary time gating changes these experimentally measured results to $31730+/-1 \%$ and $10900+/-1.67 \%$ counts respectively, by selecting only the counts in the inelastic window. The ratio of the 1779 to the 6129 line areas is now $\sim 3$,,making the experimentally derived $\mathrm{Si} / \mathrm{O}$ ratio $3 / 4.89=0.61$, which is closer to the assay concentration ratio, but is still not in good agreement.

While we have eliminated some other contributions by time gating, we have not yet removed the contributions from delayed activity (constant in time) to the peaks obtained during the inelastic window. If we make that final correction, the measured peak areas become $26273+/-1.2 \%$ and $10045+/-1.8 \%$ counts for the 1779 and $6129 \mathrm{keV}$ lines respectively, and the ratio of the $\mathrm{Si}$ to $\mathrm{O}$ peaks is now 2.5. The experimentally measured $\mathrm{Si} / \mathrm{O}$ concentration ratio is now $2.5 / 4.89=0.51$, in excellent agreement with the 0.52 value provided by the chemical assay. The dramatic improvement in the accuracy of the measured elemental concentration ratios is obtained with only minor deterioration in the percent error. The error reported for the experimentally measured peak counts, is the true precision since it is derived only from the reaction used to determine the elemental concentrations.

The use of optimized time windows allows us to obtain the most statistically precise measure of the delayed activity so that we can retain the best possible precision for the net peak counts while substantially improving on the accuracy of the measured elemental concentration. Once the counts in a peak are known to only be due to a single element and type of reaction, MCNPX calculations can accurately relate the counts to elemental concentration. It is also worth noting that the half lives of the delayed activations are typically at least 1000 times longer than the neutron period, so they can be considered to be constant during the neutron generator pulse period, as assumed in the above analysis, eliminating the need to even correct for the half lives.

\subsection{Identifying and removing sources of systematic error using TLIST} data

When working with a weak constant neutron source (e.g. from GCRs) there is no need to record event-by-event time and energy data if the data are transferred periodically with reasonable frequency, since each chunk of transferred data can be separately analyzed to identify a problem with the instrument, e.g. deteriorated resolution, and removed without compromising the entire concatenated data set. However, it is still difficult to determine if the collected data have been compromised due to other errors. These difficulties can be mitigated for the case of in situ gammaray and neutron spectroscopy measurements with the PING instrument, since it takes advantage of a pulsed neutron generator synchronized with gamma-ray and neutron detector data acquisition combined with the ability to post-process acquired time-tagged event-by-event data.

A unique benefit of incorporating a pulsed neutron generator with a time-tagged event-by-event data acquisition system is that regions in time containing suspicious data can be isolated and removed from the data set for further inspection without affecting the usefulness of the remaining data. Systematic errors in data are nearly impossible to anticipate but often can be identified when examining the post-processed data. Examples include systematic errors caused by equipment operating parameter changes, such as temperature effects on a detector response or, as illustrated in the data shown in Table 3 below, changes in the time-dependence of the turn on of neutroninduced gamma-ray flux that occurs during the PNG burst period.

We demonstrate the merit of saving event-by-event time and energy data with our analysis of the gamma-ray count rate of the $6129 \mathrm{keV}$ peak from neutron inelastic scattering on ${ }^{16} \mathrm{O}$ for a $2-\mathrm{h}$
Table 3

Fast neutron induced count rate and uncertainty for the $6129 \mathrm{keV}{ }^{16} \mathrm{O}\left(\mathrm{n}, \mathrm{n}^{\prime} \gamma\right)$ gamma ray peak for ten time slices during the PNG pulse.

\begin{tabular}{rccl}
\hline Time slice & Time range $(\mu \mathrm{s})$ & Count rate $(\mathrm{cts} / \mu \mathrm{s})$ & Uncertainty $(\mathrm{cts} / \mu \mathrm{s})$ \\
\hline 1 & $0-10$ & 9 & \pm 1 \\
2 & $10-20$ & 55 & \pm 4 \\
3 & $20-30$ & 41 & \pm 3 \\
4 & $30-40$ & 42 & \pm 3 \\
5 & $40-50$ & 39 & \pm 3 \\
6 & $50-60$ & 42 & \pm 3 \\
7 & $60-70$ & 41 & \pm 3 \\
8 & $70-80$ & 41 & \pm 3 \\
9 & $80-90$ & 46 & \pm 3 \\
10 & $90-100$ & 45 & \pm 3 \\
\hline
\end{tabular}

live time gamma-ray acquisition by the PING instrument set-up on the basalt monument. Since the neutron inelastic scattering gamma-ray production rate is proportional to the fast neutron flux, we assume that a stable gamma-ray count rate can be obtained from the time the "pulse start" signal is given to the PNG ion source $(t=0 \mu \mathrm{s})$. We can examine the time dependence of the fast neutron-induced gamma-ray flux from the time of the "pulse start" signal to the end of the PNG pulse $(t=0-100 \mu \mathrm{s})$ to look for anomalies.

In this example, we generated gamma-ray energy spectra for each of ten time slices (time slice width $=10 \mu \mathrm{s}$ ) of the gammaray data during the PNG pulse and determined the $6129 \mathrm{keV}$ net gamma-ray peak count rate and its associated uncertainty for each time slice. Table 3 lists the time range for each time slice, the $6129 \mathrm{keV}$ peak count rates and the uncertainty in the count rates for each of the ten time slices. Note that the count rates in the first and second time slices are inconsistent with the count rates in the 8 other time slices and that the count rate for these later 8 time slices is constant as expected.

The low $6129 \mathrm{keV}$ gamma-ray count rate during the first time slice $(t=0-10 \mu \mathrm{s})$ indicates that the PNG has not begun producing fast neutrons yet, since there is a delay between the time that the PNG is sent the "burst on" command signal and the time when fast neutrons are actually being generated by the PNG. The higher $6129 \mathrm{keV}$ gamma-ray count rate in the second time slice $(t=10$ $20 \mu \mathrm{s}$ ) is also inconsistent with the average value for the other slices and may be due to a systematic error induced by the gamma-ray detector electronics. In both cases, we can choose to exclude these data points from further analysis, since they are not representative of the constant inelastic gamma-ray flux during the PNG pulse. The number of neutrons produced between bursts is negligible. The PNG is designed to have a well-defined, repeatable neutron burst shape with a sharp $14.1 \mathrm{MeV}$ neutron cutoff between bursts that enables optimum timing of the inelastic and capture measurements and a capture measurement uncontaminated by inelastic gamma rays [20,21].

To be sure, we would investigate the origin of the systematic errors that prompt us to remove the data from the main analysis. Without this event-by-event time and energy data, however, these points would have been unexamined and included in the data, skewing the results. Excluding the data from the first $20 \mu \mathrm{s}$ will increase the statistical error on the mean value of the $6129 \mathrm{keV}$ gamma-ray production rate, but will result in more accurate data that we can use to infer the bulk elemental composition of planetary material. This is clearly seen by comparing the $6129 \mathrm{keV}$ weighted mean count rate and uncertainty for time slices 3 through $10(t=20-100 \mu \mathrm{s})$ which is $42.1 \mathrm{cts} /$ $\mu \mathrm{s} \pm 1.10 \mathrm{cts} / \mu \mathrm{s}$ versus the $6129 \mathrm{keV}$ weighted mean count rate and uncertainty for time slices 1 through $10(t=0-100 \mu \mathrm{s})$ which is $40.1 \mathrm{cts} / \mu \mathrm{s} \pm 0.82 \mathrm{cts} / \mu \mathrm{s}$. The difference between these two averages is two times the statistical uncertainty, resulting in a 
systematic error that would compromise the accuracy of derived elemental concentrations.

\section{Conclusions}

Many of the problems typically encountered by planetary gamma-ray elemental composition measurements are addressed by using PING in event-by-event data acquisition mode. For example, it is generally impossible to know a priori how to set optimum time windows for gamma-ray detection when using a pulsed neutron generator as the source of neutrons, because of compositional variations from location to location on a planetary body. This is a problem because there are at best only a few opportunities to acquire a specific set of data during planetary missions. This problem is solved when taking data in an event-byevent mode, optimum time windows may be set based on the data after it is collected and analyzed.

Our goal is to obtain the best estimate of elemental concentrations from the gamma-ray data. However, the same energy gamma ray can often be created from different isotopes via two different reaction mechanisms. In such instances we can separate out different time regions where a particular gamma ray is due to a specific reaction mechanism.

Post-processing event-by-event data allows PING to obtain the best precision and most accurate results. For example, in the analysis of a peak that only occurs in one time region, one can reduce its uncertainty by $\sim 40 \%$ by eliminating background in that energy region that occurs at times when the peak is not present. Perhaps even more important is the improvement in accuracy that can be achieved when the same gamma ray peak can be obtained at different times from different reaction mechanisms. The inelastic window in Table 2 for the 1779 gamma-ray peak is largely from the ${ }^{28} \mathrm{Si}\left(\mathrm{n}, \mathrm{n}^{\prime} \gamma\right)$ reaction. However this area must be corrected for the delayed activity present. The result is a factor of 3 smaller than the $1779 \mathrm{keV}$ area for the entire time spectrum, but the reduced area can now be converted to weight percent Si.

Another improvement in the accuracy of the results can be obtained by eliminating data when it appears the instrument is not performing properly as shown in Table 3 and discussed in Section 3.4. For example, the $6129 \mathrm{keV}$ weighted mean average for $0-100 \mu \mathrm{s}$ is $40.1 \mathrm{cts} / \mu \mathrm{s} \pm 0.82 \mathrm{cts} / \mu \mathrm{s}$ and for $20-100 \mu \mathrm{s}$ is $42.1 \mathrm{cts} / \mu \mathrm{s} \pm 1.10 \mathrm{cts} / \mu \mathrm{s}$. Although the statistical error of the weighted mean average increases when you exclude the first $20 \mu \mathrm{s}$, the difference between these two averages is two times the statistical uncertainty and would impact the accuracy of the derived bulk elemental concentrations of planetary material.

We can also minimize instrumental problems by subdividing the total data set at certain times to investigate such things as gain shifts. Thus by independently analyzing subsets of the data, you can preserve data quality that would be compromised if you were limited to only analyzing PHA data.

When using a pulsed neutron source, the potential exists for obtaining higher precision data. By using event-by-event data acquisition, the risk of improper timing settings is eliminated and systematic errors can be reduced or eliminated. Taken together, event-by-event data acquisition of pulsed neutron-induced gamma-ray spectra for determining elemental concentrations, provides significant enhancements to measurements obtained on a planetary surface resulting in the best scientific information on a particular mission.

\section{Acknowledgments}

We would like to thank S.L. Floyd, M. Namkung, and S.F. Nowicki for assistance with acquiring the data. We would like to thank R. Forsythe for assistance with the data analysis. We would like to thank J.I. Trombka and T.P. McClanahan for many useful discussions. We are indebted to NASA, NSF, and the Tennessee Space Grant for partial support of this research.

\section{References}

[1] W.C. Feldman, et al., Journal of Geophysical Research 109 (2004) E07S06.

[2] W.V. Boynton, et al., Space Science Reviews 110 (2004) 37.

[3] W.V. Boynton, et al., Science 297 (2002) 81.

[4] T.H. Prettyman, et al., IEEE Transactions on Nuclear Science N50 (2003) 1190

[5] J.O. Goldsten, et al., Space Science Reviews 131 (2007) 339.

[6] L.G. Evans, et al., Meteoritics and Planetary Science 36 (2001) 1639.

[7] I.G. Mitrofanov, et al., Space Science Reviews 150 (2010) 183.

[8] I.G. Mitrofanov, et al., Astrobiology 8 (2008) 793.

[9] A. Parsons, et al., Nuclear Instruments and Methods in Physics Research Section A 652 (2011) 674.

[10] A.E. Metzger, et al., Science 179 (1973) 800.

[11] L. Szentmiklosi, et al., Journal of Radioanalytical and Nuclear Chemistry 262 (2005) 213.

[12] J.A. Grau, et al., International Journal of Radiation Applications and Instrumentation Part E 7 (1993) 173.

[13] D.B. Pelowitz, et al., MNCPX User's Manual, Version 2.5.0, LANL, Los Alamos, LA-UR-05-0369, 2005.

[14] J. Bodnarik, et al., 41st LPSC, Conference Proceedings Woodlands, TX, 1-5 March, 41 (2010) 2581.

[15] P. Ann, IEEE Conference Rec. IEEE (NSS/MIC), Valencia, Spain, 23-29 October (2011) 1234, http://dx.doi.org/10.1109/NSSMIC.2011.6154608.

[16] CANBERRA Lynx Digital Signal Analyzer Application Note, (2012) <http:// www.canberra.com/literature/438222.asp $>$.

[17] J.G. Bodnarik, et al., IEEE Conference Rec. IEEE (NSS/MIC), Knoxville, TN, 30 October-6 November, (2010) 1, http://dx.doi.org/10.1109/NSSMIC.2010. 6036247.

[18] D. Burger, Personal Communication (2011).

[19] D.L. Chichester, et al., Journal of Radioanalytical and Nuclear Chemistry 271 (2007) 629.

[20] R.J. Radtke, et al., SPWLA 53rd Annual Logging Symposium, Cartagena, Columbia, June 16-20, 2012.

[21] Thermo Scientific MP320 Neutron Generator Operation Manual. 